\title{
Retinal redetachment after removal of intraocular silicone oil tamponade
}

\author{
Jost B Jonas, Harald L J Knorr, Rainer M Rank, Wido M Budde
}

Department of Ophthalmology and Eye Hospital, University Erlangen-Nürnberg, Germany

J B Jonas

H L J Knorr

R M Rank

W M Budde

Department of Ophthalmology and Eye Hospital, Faculty of Clinical Medicine Mannheim, RuprechtKarls-University

Heidelberg, 68167 Mannheim, Germany J B Jonas

Correspondence to: Dr J Jonas,

Universitäts-Augenklinik, Theodor-Kutzer-Ufer 1-3, 68167 Mannheim, Germany Jost.Jonas@

augen.ma.uni-heidelberg.de

Accepted for publication 11 April 2001

\begin{abstract}
Aim-To evaluate frequency and risk factors of retinal redetachment after removal of intraocular silicone oil tamponade.

Methods-The study included 225 patients who consecutively underwent intraocular silicone oil removal at a mean interval of 10 months after pars plana vitrectomy had been performed by one of two surgeons. Mean follow up time was 17.37 (SD 14.40) months (range 3.02-67.42 months). Results-In 57 of $225(25.3 \%)$ patients, the retina detached after removal of silicone oil. Risk factors for retinal redetachment were the following: number of previously unsuccessful retinal detachment surgeries $(p=0.0008)$; surgeon $(p=0.007)$; visual acuity before silicone oil removal $(p=0.009)$; incomplete removal of vitreous base $(p=0.01)$; absence of an encircling band in eyes with proliferate vitreoretinopathy in which an inferior retinotomy had not been performed $(p=0.01)$; and indication for pars plana vitrectomy. Rate of retinal redetachment was statistically $(p>0.05)$ independent of the technique of silicone oil removal and duration of silicone oil endotamponade.

Conclusion-Retinal redetachment after removal of silicone oil endotamponade can occur in approximately a fourth of patients, depending on the criteria to use and to remove silicone oil. Risk factors for recurrent detachment included the following: number of previously unsuccessful retinal detachment surgeries, surgeon, preoperative visual acuity, incomplete removal of the vitreous base, absence of an encircling band, and reason for pars plana vitrectomy. The rate of retinal redetachment is independent of the technique of silicone oil removal and duration of silicone oil endotamponade, with a minimal duration of silicone oil tamponade of about 3 months in the present study.

(Br f Ophthalmol 2001;85:1203-1207)
\end{abstract}

Since the development of pars plana vitrectomy by Machemer and colleagues ${ }^{1}$ for treatment of complicated retinal detachment and proliferate vitreoretinopathy, silicone oil has increasingly been used for retinal tamponade, after stiff retinal folds have been mobilised. ${ }^{2-12}$ Since silicone oil can lead to long term complications such as secondary macrophagocytic open angle glaucoma, progressive cataract, and corneal endothelial decompensation, ${ }^{13-18}$ it should be removed after adequate duration of endotamponade.
After removal of silicone oil, however, the retina can redetach. ${ }^{19-22}$ The purpose of the present study was to evaluate how often and after what duration of silicone oil tamponade the retina redetaches after silicone oil removal; which risk factors are associated with retinal redetachment; and what the visual outcome is after silicone oil removal. The answers to these questions may be useful to determine when silicone oil can be removed, whether and when additional procedures should be performed to prevent a retinal redetachment, and what visual acuity may be obtained after silicone oil removal.

\section{Patients and methods}

The study included all 225 patients who consecutively underwent pars plana vitrectomy with silicone oil endotamponade for the first time, in whom silicone oil was removed in the study period from 1994 to 1998 , and for whom data of a follow up period after silicone oil removal of at least 3 months were available (Table 1). Criteria to remove the silicone oil were a completely attached retina as determined ophthalmoscopically, and the duration of silicone oil tamponade of at least 3 months. However, in 23 of 225 patients $(10.2 \%)$, duration of silicone oil tamponade was less than 3 months. All patients were operated on by one of two surgeons (HLK and JBJ) using the same technique. The data acquisition was performed by two investigators (WMB and RMR) independently of the surgeons.

Indications for pars plana vitrectomy were proliferate diabetic retinopathy $(n=40)$, and proliferate vitreoretinopathy $(n=185)$ (Table 1). In general, silicone oil was used in eyes with diabetic retinopathy with tractional detachment of the macula including retinal defects with surrounding retinal detachment and bleeding vessels at the end of surgery. In proliferate vitreoretinopathy, silicone oil was used if there was proliferate vitreoretinopathy of grade $\mathrm{C}$ or grade $\mathrm{D}$, in eyes with giant retinal tears extending more than 90 degrees, and in highly myopic eyes with retinal detachment due to macular holes. Out of the 122 patients with proliferate vitreoretinopathy after rhegmatogenous retinal detachment (Table 1), 73 $(59.8 \%)$ patients had undergone previous, however unsuccessful, surgeries including buckling procedures before pars plana vitrectomy with silicone oil instillation was performed. Out of the 122 patients with proliferate vitreoretinopathy after rhegmatogenous retinal detachment (Table 1), 28 (23.0\%) patients showed proliferate vitreoretinopathy grade C, and $94(77.0 \%)$ patients showed proliferate vitreoretinopathy grade $\mathrm{D}$. 
Table 1 Patient and retinal characteristics (mean (SD); range)

\begin{tabular}{|c|c|}
\hline Number & 225 \\
\hline Females/males & $88 / 137$ \\
\hline Age (years) & $51.55(18.45)(4-89)$ \\
\hline Refractive error (dioptres) & $-2.55(6.33)(-25.0$ to +5.50$)$ \\
\hline Follow up time (months) & $17.37(14.40)$ (median, $12.03 ; 3.02$ to 67.42$)$ \\
\hline \multicolumn{2}{|l|}{ Indications for surgery: } \\
\hline Proliferate diabetic retinopathy & 40 \\
\hline Proliferate vitreoretinopathy (PVR) & 185 \\
\hline \multicolumn{2}{|l|}{ Because of: } \\
\hline Rhegmatogenous retinal detachment & 122 \\
\hline Penetrating ocular injuries & 30 \\
\hline $\begin{array}{l}\text { Ocular contusion with haemophthalmos and } \\
\text { retinal detachment }\end{array}$ & 13 \\
\hline $\begin{array}{l}\text { Macular hole with retinal detachment in } \\
\text { highly myopic eyes }\end{array}$ & 11 \\
\hline $\begin{array}{l}\text { Other reasons (acute postoperative infectious } \\
\text { endophthalmitis with iatrogenic retinal } \\
\text { defects; status after expulsive haemorrhage }\end{array}$ & 9 \\
\hline
\end{tabular}

All patients included in the study underwent pars plana vitrectomy using a three port technique. In all patients, silicone oil with a viscosity of 5000 centistokes was used. Pars plana vitrectomy with silicone oil endotamponade was carried out 1-83 months (mean 10.15 (SD 10.44) months; median 6.84 months) before removal of silicone oil. Depending on the intraoperative situation, additional procedures performed during pars plana vitrectomy included use of an encircling band, membrane peeling, endodrainage of subretinal fluid, use of perfluorocarbon liquids, endolaser coagulation, cryopexy, relaxing retinotomies, and cataract surgery. Selection criteria to use an encircling band were the time of surgery and the severity of proliferate vitreoretinopathy. $\mathrm{Pa}$ tients operated on at the start of the study period and patients operated on at the end of the study generally received an encircling band. Patients operated on in the middle of the study period received an encircling band if the degree of proliferate vitreoretinopathy was D2 or higher. On ophthalmoscopic examination before removal of the silicone oil, the retina was attached in all patients.

Silicone oil was removed via pars plana sclerotomies in $158(70.2 \%)$ patients and through the pupil in the remaining $67(29.8 \%)$ patients. The transpupillary approach was combined with cataract surgery, planned posterior capsulorhexis, transpupillary removal of the silicone oil, and implantation of a posterior chamber lens ${ }^{23}$ in $57(25.3 \%)$ patients. Silicone oil removal was performed under general anaesthesia in $140(62.2 \%)$ patients, in $75(33.3 \%)$ patients with retrobulbar anaesthesia, and in $10(4.4 \%)$ patients with subconjunctival anaesthesia.

\section{Results}

RETINAL REDETACHMENT

The retina remained attached after removal of silicone oil in 168 of the 225 (74.7\%) patients included in the study. In $57(25.3 \%)$ patients, the retina detached after removal of silicone oil. Three of these 57 eyes (two patients with proliferate vitreoretinopathy, one patient with proliferate diabetic retinopathy) had later to be enucleated owing to persistent retinal detachment with painful absolute secondary angle closure glaucoma.
WHOLE STUDY GROUP

Statistically significant risk factors

Fifty one of the $185(27.6 \%)$ patients from the group with proliferate vitreoretinopathy and six of $40(15.0 \%)$ patients of the group from proliferate diabetic retinopathy developed retinal redetachment. The difference in the frequency of retinal redetachment between the groups was marginally significant $\left(p=0.06 ; \chi^{2}\right)$. Comparing the various study groups with each other, rate of postoperative retinal redetachment was lowest $(p<0.05)$ in the group of patients with proliferate diabetic retinopathy and in highly myopic patients (myopic refractive error higher than -8 dioptres) operated on for macular holes with retinal detachment, followed by patients operated on for retinal giant tears with retinal detachment, and finally patients with other reasons for proliferate vitreoretinopathy.

Prevalence of an incomplete removal of the vitreous base, defined as ophthalmoscopically visible remnants of the vitreous base before removal of silicone oil, was significantly $(p<0.01)$ higher in the group of patients with retinal redetachment than in the group of patients without postoperative retinal detachment.

The frequency of retinal redetachment depended on the surgeon who had performed the pars plana vitrectomy. The retina detached significantly $(p=0.003)$ more often in patients operated on by surgeon " $A$ " compared with the patients operated on by surgeon "B." The same was found if only patients with proliferate vitreoretinopathy were taken into account $(p=0.002)$. The two subgroups each operated on by one of the two surgeons did not vary significantly in age $(p=0.20)$, sex $(p=0.34)$ and refractive error of the patients, reason for pars plana vitrectomy, duration of silicone oil endotamponade $(\mathrm{p}=0.30)$, and type of anaesthesia $(\mathrm{p}=0.41)$.

The occurrence of a retinal redetachment depended significantly on the number of retinal surgeries performed before pars plana vitrectomy with silicone oil tamponade. $\mathrm{Pa}-$ tients with retinal redetachment had undergone significantly more retinal detachment surgeries than patients with the retina remaining attached after silicone oil removal (1.55 (0.74) surgeries versus 1.33 (0.68) surgeries; $p=0.02)$.

Frequency of a relative afferent pupillary defect and frequency of a slight to marked vitreous haemorrhage in the first 3 days after silicone oil removal were significantly $(p<0.01)$ higher in patients who experienced a postoperative retinal redetachment after removal of silicone oil than in patients with their retinas remaining attached after silicone oil removal.

\section{Factors without prognostic importance}

Frequency of retinal redetachment after silicone oil removal did not vary significantly $(p>0.05)$ if the silicone oil was removed through a planned posterior capsulorhexis in combination with cataract operation and posterior chamber lens implantation than as if silicone oil was removed though pars plana 
sclerotomies $(21 / 58=36.2 \%$ versus $31 / 127=$ $24.4 \%$ ). Development of retinal redetachment was statistically independent $(p=0.51)$ of the duration of silicone oil endotamponade (10.76 (11.61) months (median, 7.00 months) versus 8.39 (6.56) months (median, 6.56 months)); the minimum duration of silicone oil endotamponade was 1 month in the present study.

\section{PROLIFERATE VITREORETINOPATHY}

Within the group of proliferate vitreoretinopathy, 148 of $185(80 \%)$ eyes had received an encircling band, and for the remaining 37 $(20 \%)$ eyes, an encircling band had not been used. Thirty eight of the $148(25.7 \%)$ eyes with an encircling band and 13 of the $37(35.1 \%)$ eyes without an encircling band developed a retinal redetachment after silicone oil removal. The difference between the two groups was marginally significant $(\mathrm{p}=0.08)$. In the group of eyes without high myopia, eyes without an encircling band developed a retinal redetachment more often than eyes with an encircling band. The relation between the absence of an encircling band and the development of a retinal redetachment was significant $(p=0.03)$ for eyes without an inferior retinotomy, but this was not significant for eyes with inferior retinotomy $(\mathrm{p}=0.60)$.

Considering only patients with proliferate vitreoretinopathy, patients with retinal redetachment after silicone oil removal and patients with the retina remaining attached after silicone oil removal did not vary significantly in age and refractive error (Table 2). Phakic patients and pseudophakic patients did not differ significantly $(p=0.27)$ in the frequency of postoperative retinal redetachment $(29 / 97=$ $29.9 \%$ versus $22 / 88=25 \%$ ). In a similar manner, highly myopic eyes with proliferate vitreoretinopathy and non-highly myopic eyes with proliferate vitreoretinopathy did not differ significantly $(p=0.58)$ in the rate of retinal redetachment.

A logistic regression model was performed, using as the dependent variable the occurrence of a retinal redetachment, and using as the independent variables preoperative visual acuity, surgeon, presence of an encircling band, presence of remnants of the vitreous base, and number of retinal detachment surgeries before the first pars plana vitrectomy with silicone oil tamponade, occurrence of a retinal redetachment was significantly associated with the number of previously unsuccessful retinal detachment surgeries before pars plana

Table 2 Patients operated on for proliferate vitreoretinopathy, divided into patients with retinal redetachment after silicone oil removal and patients with the retina attached after removal of silicone oil. Mean (SD)

\begin{tabular}{llll}
\hline & $\begin{array}{l}\text { Retinal redetachment } \\
\text { after silicone oil removal }\end{array}$ & Retina attached & p Value \\
\hline Number & 51 & 134 & \\
Right eyes/left eyes & $32 / 19$ & $67 / 67$ & 0.08 (NS) \\
Age (years) & $53.71(19.37)$ & $50.12(19.49)$ & 0.20 (NS) \\
Median & 58.0 & 53 & \\
Range & 6 to 86 & 4 to 89 & 0.95 (NS) \\
Refractive error (D) & $-4.28(7.18)$ & $-3.86(5.96)$ & \\
Median & -1.50 & -1.13 & \\
Range & -25.0 to +5.5 & -22.0 to +5.0 & \\
\hline
\end{tabular}

NS = statistically not significant.
Table 3 Visual acuity in the whole study group (mean (SD)

\begin{tabular}{ll}
\hline Before pars plana vitrectomy & $0.11(0.19)$ \\
Median & 0.03 \\
Range & Light perception to 1.0 \\
After pars plana vitrectomy with & \\
silicone oil endotamponade & $0.15(0.18)$ \\
Median & 0.08 \\
Range & Light perception to 1.25 \\
After removal of silicone oil & $0.17(0.20)$ \\
Median & 0.10 \\
Range & No light perception to 1.0 \\
Visual acuity $>0.02$ & $81 \%$ \\
Visual acuity $>0.05$ & $63 \%$ \\
Visual acuity $>0.10$ & $35 \%$ \\
Visual acuity $>0.20$ & $11 \%$ \\
Visual acuity $>0.50$ & $7 \%$ \\
\hline
\end{tabular}

vitrectomy with silicone oil tamponade $(\mathrm{p}=0.0008)$, surgeon $(\mathrm{p}=0.007)$, poor preoperative visual acuity before silicone oil removal $(p=0.009)$, presence of vitreous base remnants $(\mathrm{p}=0.01)$, and absence of an encircling band $(p=0.01)$. In the same multivariate logistic regression analysis, presence or absence of the native lens as independent factor was not significantly $(p=0.66)$ associated with the rate of retinal redetachment after silicone oil removal.

VISUAL ACUITY

The group with proliferate vitreoretinopathy and the group with proliferate diabetic retinopathy did not differ significantly $(p=0.48)$ in visual acuity before pars plana vitrectomy (0.12 (0.21) versus $0.06(0.11))$ (Table 3)). After silicone oil instillation, visual acuity was significantly better in eyes operated on for proliferate vitreoretinopathy compared with eyes operated on for proliferate diabetic retinopathy (0.16 (0.18) versus $0.10(0.16) ; \mathrm{p}=0.004)$. After removal of silicone oil, visual acuity remained significantly better for the patients operated on for proliferate vitreoretinopathy than for the patients with proliferate diabetic retinopathy $(0.18(0.22)$ versus $0.11(0.14)$; $\mathrm{p}=0.029)$.

The frequency of retinal redetachment after silicone oil removal depended significantly on visual acuity before silicone oil removal $(p=0.004)$ and on visual acuity in the postoperative period after silicone oil removal $(p<0.001)$. The same hold true if only patients with proliferate vitreoretinopathy were taken into account $(p=0.003$ and $p<0.001)$. In the group of patients with diabetic proliferate retinopathy, the correlation was not significant $(\mathrm{p}=0.46)$.

\section{Discussion}

In the present study, frequency of retinal redetachment after removal of silicone oil was $27.6 \%$ in patients operated on for proliferate vitreoretinopathy, and it was $15.0 \%$ in patients operated on for proliferate diabetic retinopathy. These figures are comparable with those reported previously in the literature. $^{9-12} 14 \quad 16$ 19-29

\section{PROGNOSTIC RISK FACTORS}

The rate of retinal redetachment after removal of silicone oil was significantly lower in patients operated on for diabetic tractional detachment 
than in the patients treated for proliferate vitreoretinopathy. This difference which has already been described in previous studies ${ }^{20} 21$ may be explained by physiological differences between diabetic eyes and non-diabetic eyes undergoing vitrectomy. In diabetic retinopathy compared with proliferate vitreoretinopathy, the proliferate process is usually confined to the paracentral and mid-peripheral retina, and pre-existing or iatrogenic retinal breaks tend to be more posterior and thus more easily accessible to surgical relief of any associated vitreoretinal traction.

The finding of vitreous remnants being an important risk factor implies clinically that, during pars plana vitrectomy, one should intensively try to remove the vitreous base, especially in the region of peripheral retinal defects. One may infer that patients with remnants of the vitreous base are poor candidates for removal of silicone oil unless additional procedures, such as re-pars plana vitrectomy for removal of the remaining vitreous, are performed. Vitreous remnants in the periphery of the fundus may exert a traction on the retina which may only be compensated as long as the internal silicone oil tamponade presses the retina against the retinal pigment epithelium.

Important postoperative factors indicating retinal redetachment were postoperative vitreous haemorrhage and presence of a relative afferent pupillary defect. The latter finding shows the high clinical importance of performing the swinging flashlight test in patients in whom, owing to an opacity of the optic media, the retina can not clearly be visualised. The association of a postoperative haemorrhage with an increased rate of retinal redetachment suggests that these patients should carefully be followed up to prevent that a re-pars plana vitrectomy is performed unnecessarily late.

In the group of proliferate vitreoretinopathy, postoperative retinal redetachment occurred significantly more often in eyes without an encircling band and without an inferior retinotomy than in eyes with an encircling band or in eyes without an encircling band in which, however, an inferior retinotomy had been performed. The use of the encircling band was not randomly distributed between the patients of the present study indicating a flaw in the study design. Since, however, an encircling band was mainly used in apparently difficult situations in the present study, one may infer that an encircling band is helpful in reducing the rate of postoperative retinal redetachments in eyes with proliferate vitreoretinopathy. The absence of an encircling band in eyes without inferior retinotomy may predispose to a retinal redetachment since a circumferential buckling of the equator may release a slight traction of remnants of the vitreous base. If the retina is no longer in direct contact with the vitreous base in eyes with an inferior retinotomy, contracting remnants of the vitreous base may no longer be able to exert a direct traction on the retina.

The number of retinal surgeries performed before pars plana vitrectomy with silicone oil tamponade was significantly associated with the occurrence of a retinal redetachment after silicone oil removal. It reflects the clinical experience that the risk of retinal redetachment depends on the grade of proliferate vitreoretinopathy which may partially depend on the number of previously unsuccessful operations. It may be taken as a hint that, in some clinical situations, primary pars plana vitrectomy may be preferred to scleral buckling procedures, and that one should not unnecessarily delay pars plana vitrectomy.

The surgeon was one of the major factors influencing the final outcome in the present studies. Although it may be true for all surgical procedures, the result was astonishing since similar or same techniques and instruments were used and since the surgical experience of the two surgeons was comparable. The dependence of a surgical result on the surgeon may be one of the reasons why studies on surgical outcome can difficult to compare with each other.

FACTORS WITHOUT INFLUENCE ON RETINAL REDETACHMENT

With a minimal time period between pars plana vitrectomy and removal of silicone oil of 1 month (usually longer than 3 months), duration of intraocular silicone oil tamponade had no significant $(\mathrm{p}=0.51)$ effect on the rate of postoperative retinal redetachment. It suggests that the retinal situation may be settled after about 1-3 months. After this time, silicone oil may be removed or, if epiretinal membranes have reformed, re-pars plana vitrectomy may be considered. It may also be true in view of histological findings showing microscopic silicone oil related changes in the retina occurring after 4 weeks of silicone oil tamponade. ${ }^{30}$

The rate of retinal redetachment after silicone oil removal was independent of the technique of silicone removal. The frequency of retinal redetachment did not differ significantly between the patients in whom the silicone oil was removed through a planned posterior capsulorhexis as part of cataract surgery, and patients in whom the silicone oil was removed through pars plana sclerotomies. As already pointed out in previous studies, ${ }^{23}$ it suggests that silicone oil may be removed through the pupil if additional vitreoretinal procedures, such as removal of vitreous remnants or epiretinal membrane, do not have to be carried out.

\section{STUDY LIMITATIONS}

There are limitations of the present study. Since it is a clinical study on the outcome of a surgical procedure, there are numerous factors influencing the results. They may lead to pseudo-significant difference and may cover significant differences between study groups. To reduce the influence of external factors, the study included only patients in whom pars plana vitrectomy and removal of silicone oil had been performed by one of two surgeons. Another limitation of a study like the present one is that the rate of postoperative retinal redetachments is markedly influenced by the criteria to perform pars plana vitrectomy and the criteria to use silicone oil. It can make it 
difficult to compare studies on the same topic. The main goals of the present study were, however, not the assessment of the rate of retinal redetachments after removal of intraocular silicone oil. The main purpose was to evaluate the risk factors leading to, and to examine the factors indicating, retinal redetachment. These figures, compared with the overall rate of retinal redetachment, may be less influenced by the criteria to use and to remove silicone oil. A further important limitation of the study is that it is a retrospective non-comparative interventional case series investigation, so that the patients were not randomly distributed between the various parameters tested in the study. It limits the generalisability of the results. For some variables, however, the non-randomised study design served only to support the findings. Although an encircling band was more often used in apparently difficult situations than in simple situations, the presence of an encircling band in eyes without inferior retinotomy was associated with a lower rate of retinal redetachment.

VISUAL ACUITY

The eventual visual outcome in the patients included in the present study was relatively encouraging (Table 3). However, one has to take into account that, after silicone oil removal, only $35 \%$ of the patients achieved a visual acuity of more than 0.10 , and that in less than $15 \%$ of the patients, final visual acuity was higher than 0.20 . These figures may suggest that when discussing pars plana vitrectomy with the patient, one should take into account the general condition of the patient and the benefit a potentially successful surgery may have on the quality of life of the patient.

Proprietary interest: none.

1 Machemer R. Proliferate vitreoretinopathy (PVR): a personal approach of its pathogenesis and treatment. Proctor Lecture. Invest Ophthalmol Vis Sci 1988;29:1771-83.

2 McCuen BW, Landers BW, Machemer R. The use of silicone oil following failed vitrectomy for retinal detachment with advanced proliferate vitreoretinopathy. Ophthalmology 1985;92:1029-34.

3 Gonvers M. Temporary silicone oil tamponade in the management of retinal detachment with proliferate vitreoretinopathy. Am 7 Ophthalmol 1985:100:239-45.

4 Dimopoulos S, Heimann K. Spät-Komplikationen nach Silikonölinjektion. Langzeitbeobachtung an 100 Fällen. Klin Monatsbl Augenheilkd 1986;189:223-2.

5 Laqua H, Lucke K, Foerster M. Results of silicone oil Laqua H, Lucke K, Foerster M. Results
surgery. fpn f Ophthalmol 1987;31:124-31.

6 Surgery. Fpn f Ophthalmol 1987;31:124-31. complicated retinal detachments. Ophthalmology 1987;94: complicated
7 Brourman ND, Blumenkranz MS, Cox MS, et al. Silicone oil for treatment of severe proliferate diabetic retinopathy. Ophthalmology 1989;96:759-64.

8 Gabel VP, Beck P. Verbessert Silikonöl die Prognose bei schwerer proliferativer diabetischer Retinopathie ? Klin Monatsbl Augenheilkd 1990;197:112-7.

9 Lucke K, Laqua H. Silicone oil in the treatment of complicated retinal detachments: techniques, results, and complications. Berlin, New York: Springer-Verlag, 1990;39-78.

10 Blumenkranz M, Azen SP, Aaberg T, et al. Relaxing retinotomy with silicone oil or long-acting gas in eyes with severe proliferate vitreoretinopathy: Silicone Study Report 5. Am f Ophthalmol 1993;116:557-64.

11 Abrams GW, Azen SP, McCuen BW II, et al. Vitrectomy with silicone oil or long-acting gas in eyes with severe proliferate vitreoretinopathy: results of additional and longterm follow-up. Silicone Study Report II. Arch Ophthalmol term follow-up. Silc

12 Azen SP, Scott IU, Flynn HW, et al. Silicone oil in the repair of complex retinal detachments. A prospective observational multicenter study. Ophthalmology 1998;105:158797.

13 Abrams GW, Azen SP, Barr CC, et al. The incidence of corneal abnormalities in the silicone Study. Arch Ophthalmol 1995;113:764-9.

14 McCuen BW II, de Juan E Jr, Landers MB III, et al. Silicone oil in vitreoretinal surgery. Part 2: results and complications. Retina 1985;5:198-205.

15 Burk LL, Shields MB, Proia D, et al. Intraocular pressure following intravitreal silicone oil injection. Ophthalmic Surg 1988;19:565-9.

16 Federman JL, Schubert HD. Complications associated with the use of silicone oil in 150 eyes after retina-vitreous surgery. Ophthalmology 1988;95:870-6.

17 Nguyen QH, Lloyd MA, Heuer DK, et al. Incidence and management of glaucoma after intravitreal silicone oil injection for complicated retinal detachments. Ophthalmology 1992;99:1520-6.

18 Barr CC, Lai MY, Lean JS, et al. Postoperative intraocular pressure abnormalities in the Silicone Study. Silicone study report 4. Ophthalmology 1993;100:1629-35.

19 Casswell AG, Gregor ZJ. Silicone oil removal. I. The effect on the complications of silicone oil. Br f Ophthalmol 1987; 71:893-7.

20 Casswell AG. Gregor ZJ. Silicone oil removal, II: operative and postoperative complications. Br F Ophthalmol 1987;71: 898-902.

21 Zilis JD, McCuen BW II, deJuan E, et al. Results of silicone oil removal in advanced proliferate vitreoretinopathy. $A m \mathcal{F}$ Ophthalmol 1989;108:15-21.

22 Hutton WL, Azen SP, Blumenkranz MS, et al. The effects of silicone oil removal. Silicone Study Report 6. Arch Ophthalmol 1994;112:778-85

23 Jonas JB, Budde WM, Panda-Jonas S. Cataract surgery combined with transpupillary silicone oil removal through planned posterior capsulotomy. Ophthalmology 1998;105: 1234-8.

24 Rice TA, Michels RG. Long-term anatomic and functional results for diabetic vitrectomy. Am $\mathcal{F}$ Ophthalmol 1980;90: 297-303.

25 Blankenship GW, Machemer R. Long term diabetic vitrectomy results. Report on a 10 year follow-up. Ophthalmology 1985;92:503-6.

26 Cockerham WD, Schepens CL, Freeman HM. Silicone injection in retinal detachment. Arch Ophthalmol 1970;83: $704-12$

27 Gonvers $M$. Temporary use of intraocular silicone oil in the treatment of detachment with massive periretinal proliferation. Ophthalmologica 1982;184:210-8.

28 Michels RG. Vitrectomy for complications of diabetic retinopathy. Arch Ophthalmol 1978;96:444-52.

29 Wilson-Holt N, Gregor Z. Spontaneous relieving retinotomies in diabetic silicone filled eyes. Eye 1992, 6:161-4.

30 Knorr HLJ, Seltsam A, Holbach L, et al. Intraocular silicione oil: a clinicopathological study of 36 enucleated eyes. Ophthalmologe 1996;93:130-8. 